\title{
Los emprendedores de la zona 3 del Ecuador: un análisis comparativo desde sus competencias
}

\author{
URL: http://revistas.uta.edu.ec/erevista/index.php/bcoyu/article/view/685 DOI: http://dx.doi.org/10.31164/bcoyu.20.2019.685
}

\section{Mireya Montenegro-Espinoza'; Sisa Vega-Quindigalle²; Lorena Rivera-Badillo ${ }^{3}$}

Fecha de recepción: 11 de enero de 2019

Fecha de aceptación: 12 de febrero de 2019

\section{Resumen}

El objetivo de esta investigación es realizar un análisis comparativo del perfil del emprendedor a través de sus competencias en la zona tres del Ecuador. El análisis es de tipo descriptivo, cuantitativo y cualitativo. Se aplica una encuesta a 397 emprendedores. Sus resultados se fundamentan estadísticamente con: Kaiser-Meyer-Olkin y la prueba de Bartlett; correlaciones y análisis de componentes principales, para concluir que este emprendedor es un individuo innovador, comprometido con su empresa y atento a las oportunidades, líder y comunicativo, con firmes valores éticos y morales, capaz de enfrentar dificultades de su entorno, principalmente los económicos, tecnológicos, culturales y sociales.

Palabras clave: Emprendedor, emprendimiento, competencias

\section{Abstract}

The objective of this research is to carry out a comparative analysis of the profile of the entrepreneur through his competencies in zone three of Ecuador. The analysis is descriptive, quantitative and qualitative. A survey is applied to 397 entrepreneurs. Their results are statistically based on: Kaiser-Meyer-Olkin and the Bartlett test; correlations and analysis of main components, to conclude that this entrepreneur is an innovative individual, committed to his company and attentive to opportunities, leader and communicative, with strong ethical and moral values, capable of facing difficulties of his environment, mainly economic, technological, cultural and social.

Keywords: Entrepeneur, entrepreneurship, competitions.

\section{Introducción}

La presente investigación se fundamenta en una revisión bibliográfica de 65 autores de las áreas de psicología y administración, correspondiente a los últimos 5 años, a partir de quienes se seleccionó las competencias del emprendedor como motivo de estudio. Se propone la agrupación en 4 dimensiones y un grupo de factores externos, las provincias para el estudio se encuentran ubicadas en el centro del Ecuador, catalogada por la SENPLADES ${ }^{4}$ como Zona 3 y las conforman: Cotopaxi, Chimborazo, Pastaza y Tungurahua, de esta zona se consideran las microempresas de las capitales de cada una de las provincias dentro de las 14 actividades económicas, donde se pretende conocer ¿Cuáles son las competencias que influyen en los individuos al momento de emprender en la zona 3 ?

Mediante la aplicación de una encuesta validada por expertos se conoce la importancia e influencia que tienen las 4 dimensiones referentes a las competencias esenciales de un emprendedor y un grupo de factores que estos individuos deben enfrentar (Rivera, 2017).

Para que un emprendimiento sea factible se requiere de un gestor y protagonista de la creación de una empresa, a quien la literatura especializada lo llama emprendedor. La identificación constante de las oportunidades latentes del mercado le permite ofrecer productos o servicios con el objetivo de solucionar problemas de su contexto.

Tras la revisión bibliográfica de varios especialistas del quinquenio previo a la realización de esta investigación, las autoras proponen una definición de emprendedor que se muestra en la tabla 1.
Tabla 1. Definiciones de emprendedor, según autores

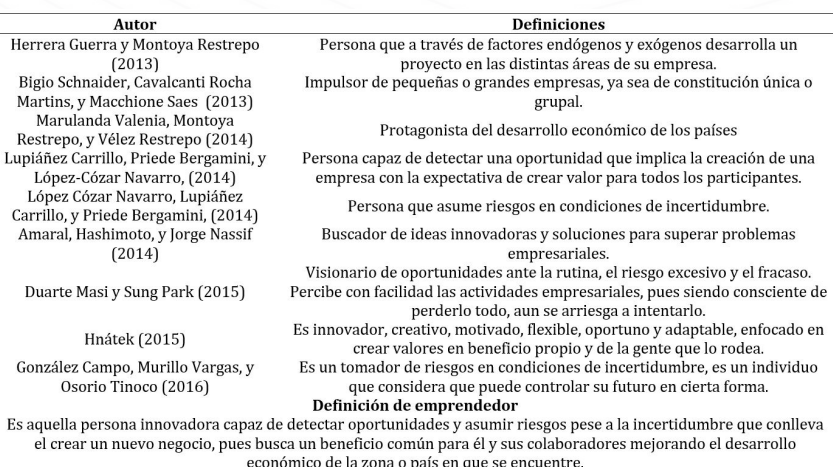
económico de la zona o país en que se encuentre.

Fuente: Elaboración propia a partir de varios autores (2013 al 2016)

Las características, aptitudes y habilidades del emprendedor lo convierten en un individuo con capacidad de promover cambios continuos, arriesgado, con liderazgo, resolutivo bajo presión, negociador, entre otras (Guzmán Barquet, Sopó Montero, Salazar Raymond, \& Vera Salas, 2017).

Con fundamento bibliográfico se identifica aquellas características que tienen en común los autores analizados, para manifestar que un emprendedor debe poseer: Innovación, logro, trabajo en equipo, liderazgo, tolerancia a la ambigüedad, control, creatividad, toma de riesgos, orientación a objetivos, autonomía y motivación (Balbastre, Canet, Escribá y Ugalde (2014); Edgerton y Roberts (2014); Hidalgo (2014); López, Lujano, y Ortega (2015); Da Silva, Domínguez y Vier (2016); Restrepo, Tapasco y Vidarte (2016); Berríos (2017); Florez, Hernández, Sánchez y Ward (2017); Guzmán, Sopó, Salazar y Vera (2017).

El perfil del emprendedor puede establecerse a partir de varios atributos como: edad, nivel educativo, condición económica, posición social y género (Restrepo Salgado, Tapasco Triviño, \& Vidarte Claros, 2016), y su actividad es motivada por necesidad u oportunidad. Adicionalmente este individuo debe poseer características que definen la estructura de su perfil basados en tres ejes fundamentales: saber ser, saber hacer, y, saber conocer (Bonilla y García Restrepo, 2014; Alvarado, Rangel, y Sierra, 2016; Gómez et al, 2017).

Esta investigación propone la agrupación por competencias a partir de análisis previo realizado a las características que son parte de la figura emprendedora (López Gómez, 2016; Viloria, 2017), que propician y mantienen su actividad pudiendo adquirirlas o construirlas mediante sus experiencias y prácticas de aprendizaje (Florez , Hernández, Sánchez García , \& Ward, 2017).

\section{Primera Dimensión: competencias psicológicas individuales}

Su análisis se centra en las características personales (Duarte Masi \& Sung Park, 2015) mediante las cuales el emprendedor identifica oportunidades empresariales (Florez et al, 2017).

La Psicología individual conocida como la teoría de Adler, considera que los seres humanos tienen una orientación natural al logro como medio para conseguir su propia autodefinición. Esta tendencia es el resultado entre el efecto y las fuerzas sociales que todo individuo enfrenta

\footnotetext{
"Universidad Técnica de Ambato. Facultad de Ciencias Administrativas/Organización de Empresas. Ambato - Ecuador. E-mail: miyesmeralda21@gmail.com. ORCID: https://orcid.org/0000-0002-3591-0704 ¿Universidad Técnica de Ambato. Facultad de Ciencias Administrativas/Organización de Empresas. Ambato - Ecuador. E-mail: sisa_05_1995@hotmail.com. ORCID: https://orcid.org/0000-0003-3364-4489 ${ }^{3}$ Universidad Técnica de Ambato. Facultad de Ciencias Administrativas/Organización de Empresas. Ambato - Ecuador. E-mail: pl.rivera@uta.edu.ec. ORCID: https://orcid.org/0000-0001-6703-2160 ${ }^{4}$ Secretaría Nacional de Planificación y Desarrollo (2018)
} 
a lo largo de su vida a través de "los retos, las metas, la perseverancia, el compromiso, el logro, el cumplimiento" (Gil Gaytán, 2017, pág. 11). En la tabla 2, se expone un resumen de las características psicológicas individuales del emprendedor.

Tabla 2. Base Teórica para identificar competencias psicológicas individuales del emprendedor

\begin{tabular}{|c|c|}
\hline Autor/Año & Rasgos psicológicos individuales \\
\hline $\begin{array}{l}\text { Herrera (2012); Gutiérrez (2015); Martínez (2016); Contreras et al } \\
\text { (2017); Berríos (2017) }\end{array}$ & $\begin{array}{l}\text { Propensión al riesgo, asumir riesgos, riesgos, } \\
\text { tolerancia al riesgo, aceptar y asumir riesgos. }\end{array}$ \\
\hline $\begin{array}{l}\text { Ahumada (2013); Ferreira y Jolonch (2015); Fuentelsaz y Montero } \\
\text { (2015); Contreras et al (2017); Florez et al (2017). }\end{array}$ & $\begin{array}{l}\text { Autoeficacia, Autocontrol, autonomía, } \\
\text { Autodisciplina, confianza en si mismo, } \\
\text { independencia de juicio }\end{array}$ \\
\hline $\begin{array}{l}\text { Lizote, Silveira y Verdinelli (2013); Amaral, Hashimoto y Nassif (2014); } \\
\text { CAF Banco de desarrollo de América Latina (2016); Berrios (2017); }\end{array}$ & Compromiso, compromiso empresarial \\
\hline $\begin{array}{l}\text { Ahumada (2013); Ferreira y Jolonch (2015); Florez et al (2017); } \\
\text { Martinez (2016); Pedrosa y Suárez (2016). }\end{array}$ & $\begin{array}{l}\text { locus de control interno; control interno, control de } \\
\text { la hostilidad }\end{array}$ \\
\hline $\begin{array}{c}\text { Herrera (2012); Álvarez, Pellicer y Torrejón (2013); Edgerton y } \\
\text { Robert (2014); Duarte y Sung Park (2015); Martínez (2016). } \\
\text { Álvarez et al (2013); Ferreira y Jolonch (2015); Martínez (2016); } \\
\text { Pedrosa y Suárez (2016). } \\
\text { Herrera (2012); Amaral et al (2014); Fuentelsaz y Montero (2015); Dos } \\
\text { Santos et al (2016) }\end{array}$ & $\begin{array}{c}\text { Capacidad de innovación; Iniciativa, Creatividad; } \\
\text { innovación, innovador, disposición a la innovación } \\
\text { motivación al logro, necesidad de logro, orientación } \\
\text { al logro } \\
\text { Estado de alerta ante oportunidades; descubrir } \\
\text { oportunidades, oportunidades rentables. }\end{array}$ \\
\hline $\begin{array}{c}\text { Duarte y Sung Park (2015); López, Lujano y Ortega (2015); Godelieve y } \\
\text { Panagiotis (2016); Berríos (2017) }\end{array}$ & Tolerancia a la ambigüedad \\
\hline
\end{tabular}

Fuente: Elaboración propia a partir de varios autores (2012 al 2017)

\section{Segunda dimensión: Competencias Psicológicas Colectivas}

Un emprendedor se relaciona con el medio que lo rodea a través de la interrelación, la capacidad de comunicación, liderazgo, espíritu de equipo, capacidad de negociación y planificación. La competencia colectiva busca la complementariedad y el encadenamiento de competencias [individuales] por medio de su personalidad, temperamento y características de modo que la relación con los actores de la cadena, sea eficiente (Climént Bonilla, 2017).

En la tabla 3 se pone de manifiesto los diferentes rasgos o características psicológicas de interrelación planteados por autores de los últimos cinco años.

Tabla 3. Base Teórica para identificar competencias psicológicas colectivas del emprendedor

\begin{tabular}{|c|c|}
\hline Autor/Año & Rasgos psicológicos colectivos \\
\hline $\begin{array}{l}\text { Alderete (2013); Alsinet, Paris, Torrelles y Sabrià (2015) De } \\
\text { Souza y Goncalves (2016); Berrios (2017) }\end{array}$ & $\begin{array}{l}\text { Capacidad de comunicación, capacidad de vínculos y relaciones } \\
\text { de confianza, comunicación empresarial, relaciones sociales. }\end{array}$ \\
\hline $\begin{array}{l}\text { Nandram, Orhei y Vinke (2015); Alsinet et al. (2015); CAF } \\
\text { Banco de desarrollo de América Latina (2016); Berríos (2017) }\end{array}$ & $\begin{array}{l}\text { Resolución de conflictos. Creación de redes remando en la } \\
\text { misma dirección. Capacidad de relaciones Humanas }\end{array}$ \\
\hline $\begin{array}{l}\text { Ahumada (2013); Castañeda, González, y Rubio (2015); } \\
\text { Godelieve y Panagiotis (2016); Berrios (2017) }\end{array}$ & $\begin{array}{l}\text { Colaboración, trabajo en equipo. Trabajar con otros. } \\
\text { Compromiso }\end{array}$ \\
\hline $\begin{array}{l}\text { Castañeda et al. (2015); CAF Banco de desarrollo de América } \\
\text { Latina (2016); Pedrosa y Suárez Âlvarez (2016) }\end{array}$ & Liderazgo. Innovador. Fe en ideas propias. \\
\hline Florez et al (2017); Berríos (2017) & $\begin{array}{l}\text { Control percibido, norma subjetiva y fiabilidad, fijación de } \\
\text { objetivos. }\end{array}$ \\
\hline
\end{tabular}

Fuente: Elaboración propia a partir de varios autores (2013 al 2017)

\section{Tercera dimensión: Competencias Cognitivas}

Las habilidades cognitivas una vez que son adquiridas entran en un ciclo de desarrollo y mejoramiento continuo que se perfeccionan para generar nuevos conocimientos. Son definidas como destrezas y procesos de la mente necesarias para realizar, adquirir y recuperar los conocimientos que se usan en varias oportunidades. Al ser innatas, pueden o no cambiar con el tiempo (Romero y Tapia, 2014).

Las competencias cognitivas incluyen procesos mentales tales como: observar, recordar, ordenar, analizar, evaluar, entre otros, y determinan la construcción de significados de forma, cualitativa y cuantitativa (Capilla, 2016).

Estas competencias requieren de la actualización constante de los conocimientos, a través de: cursos, seminarios, charlas, congresos y, otros disponibles en el entorno académico. En la tabla 4 se presentan los rasgos que forman parte de esta dimensión.

Tabla 4. Base Teórica para identificar competencias cognitivas del emprendedor

\begin{tabular}{|c|c|}
\hline Autor/Año & Competencias Cognitivas \\
\hline $\begin{array}{l}\text { Pedrosa y Suárez (2013); Alderete (2013); Feitó, } \\
\text { Mungaray y Texis-Flores (2016); Gámez, Saiz y Gil } \\
\text { (2016). }\end{array}$ & $\begin{array}{l}\text { Conocimientos tácitos y explíitos, capacidad de construcción } \\
\text { del mismo conocimiento. Conocimiento útil y especializado. }\end{array}$ \\
\hline $\begin{array}{l}\text { Meza (2013); Fuentelsaz y Montero (2015); Hochsztain y } \\
\text { Messina (2015); Martinez (2016); Jiménez (2017). }\end{array}$ & $\begin{array}{l}\text { Educación formal, nivel educativo, experiencia laboral o } \\
\text { emprendedora, redes de contactos, alta formación profesional. }\end{array}$ \\
\hline $\begin{array}{l}\text { Meza (2013); Morales y Pineda (2015); Godelieve y } \\
\text { Panagiotis (2016); Berríos (2017); Jiménez (2017). }\end{array}$ & $\begin{array}{l}\text { Educación informal, experiencia, actividades cotidianas } \\
\text { relacionadas con el trabajo. }\end{array}$ \\
\hline $\begin{array}{l}\text { Davidsson, obschonka, Stuetzer y Schmitt-Rodermund } \\
\text { (2013); Dorado, Pined, y Morales (2014); Morales y } \\
\text { Pineda (2015); Martinez (2016); Parra (2017). }\end{array}$ & $\begin{array}{l}\text { Experiencia previa, educación, experiencia laboral previa, } \\
\text { capital humano, capacidad de pensamiento. }\end{array}$ \\
\hline
\end{tabular}

Fuente: Elaboración propia a partir de varios autores (2013 al 2017)

\section{Cuarta dimensión: Competencias Axiológicas}

Los emprendedores a más de tener características distintivas pueden incluir en sus actividades elementos axiológicos como: creencias, valores y ética, para promover bienestar económico y social. Las creencias se construyen en el transcurso de la vida y ayudan a establecer parámetros de pensar y actuar (Rivera, 2017). Los valores determinan la actitud de las personas hacia la sociedad y la naturaleza. La ética es el estudio de los valores morales y la conducta de los individuos (Chaturvedi, 2014; Gordienko et al., 2017). En la tabla 5 se establece las características que forman parte de cada uno de los elementos de las competencias axiológicas.

Tabla 5. Base Teórica para identificar competencias axiológicas del emprendedor

\begin{tabular}{cc}
\hline Autor/ Año & Competencias Axiológicas \\
\hline $\begin{array}{c}\text { Hidalgo (2014); Bernal et al (2014); De Azevedo et al (2015); Benatti } \\
\text { y Saltorato (2016); Rodionova y Shvachkina (2018) }\end{array}$ & Creencias, hábitos, costumbres. \\
Hidalgo (2014); Dion (2014); Albán et al (2017); Berríos (2017); \\
$\begin{array}{c}\text { Rodionova y Shvachkina (2018). } \\
\text { Valores, altruismo, creencias, ideologías. }\end{array}$ \\
$\begin{array}{c}\text { Dion (2014); López et al (2017); Rodionova y Shvachkina (2018) } \\
\text { Ética, responsabilidad social, cuidado del medio } \\
\text { ambiente, equidad. }\end{array}$ \\
\hline
\end{tabular}

\section{Factores del entorno}

Estos factores se refieren a todo evento fuera de la empresa que puede afectarla o no, estos elementos se agrupan en siete segmentos del entorno: demográficos, económicos, político-legal, sociocultural, tecnológico, global y físico (Ina Primiana, 2015). De los siete elementos mencionados se dará principal énfasis a los cinco primeros que se presenta en la tabla 6 de una manera más detallada.

Tabla 6. Base teórica para identificar los factores externos que influyen en la actividad emprendedora

\begin{tabular}{|c|c|}
\hline Autor/Año & Factores del entorno \\
\hline $\begin{array}{l}\text { Herrera y Montoya (2013); Hidalgo (2014); López, Lupiáñez y } \\
\text { Priede (2014); Berríos (2017); Criado y otros (2017). }\end{array}$ & $\begin{array}{l}\text { Económicos, generadora de recursos, conocimiento de } \\
\text { negocios, sentido numérico, administrar recursos. }\end{array}$ \\
\hline $\begin{array}{l}\text { Caetano, Curral y Santos (2013); Biedma, Martínez y Ruiz } \\
\text { (2015); Klein (2016); Dos Santos, Gazolo, Moreno y Vier } \\
\text { (2016). }\end{array}$ & $\begin{array}{l}\text { Políticos, políticas gubernamentales, político-legal, } \\
\text { política pública }\end{array}$ \\
\hline $\begin{array}{l}\text { Herrera y Montoya (2013); López, Lupiáñez y Priede (2014); } \\
\text { Biedma, Martínez, \& Ruiz (2015); Montiel y Palma (2016). }\end{array}$ & $\begin{array}{l}\text { Tecnológicos, investigación y desarrollo, innovación, } \\
\text { avances tecnológicos. }\end{array}$ \\
\hline $\begin{array}{l}\text { Apetrei et al (2013); Hidalgo (2014); Dos Santos et al (2016); } \\
\text { De Souza y Martines (2017); Rodionova y Shvachkina (2018). }\end{array}$ & $\begin{array}{l}\text { Culturales, conocimiento, creencias, moral, leyes, } \\
\text { costumbres, herencias culturales, normas, tradiciones }\end{array}$ \\
\hline $\begin{array}{l}\text { Herrera y Montoya (2013); Hidalgo (2014); Hochsztain y } \\
\text { Messina (2015); Biedma et al (2015); Berríos (2017). }\end{array}$ & $\begin{array}{l}\text { Sociales, redes de contactos, so de personas de } \\
\text { recursos externos. }\end{array}$ \\
\hline
\end{tabular}

Fuente: Elaboración propia a partir de varios autores (2013 al 2018)

\section{Metodología}

La presente investigación se apoya en un estudio descriptivo con método cuantitativo-cualitativo. Como instrumento se aplicó un cuestionario tipo encuesta validado por expertos en emprendimiento, dicha validación fue realizada en la tesis doctoral "El emprendimiento y la creación de empresa. Etapas y factores clave de éxito" (Rivera Badillo, 2016, pág. 193), investigación ejecutada en una de las provincias que forma parte de la zona 3. Para efectos de este estudio se tuvo la autorización de la autora para incluir en la herramienta las 12 actividades económicas que generan desarrollo económico de la zona.

La estructura de la encuesta tiene cinco variables representadas en la tabla 7.

\begin{tabular}{|c|c|c|}
\hline Variables & Número & items \\
\hline $\begin{array}{l}\text { Competencias psicológicas } \\
\text { Individuales }\end{array}$ & (1) & $\begin{array}{c}\text { Asumir riesgos, autonomía, compromiso, control interno, innovación, } \\
\text { motivación al logro, orientación a las oportunidades y tolerancia a la } \\
\text { ambigüedad. }\end{array}$ \\
\hline $\begin{array}{l}\text { Competencias psicológicas } \\
\text { colectivas }\end{array}$ & 5 & $\begin{array}{l}\text { Capacidad de comunicación, capacidad de negociación, espíritu de equipo, } \\
\text { liderazgo y planificación. }\end{array}$ \\
\hline Competencias cognitivas & 4 & $\begin{array}{l}\text { Conocimiento en el área especifico del negocio, educación formal, } \\
\text { educación informal y experiencia previa. }\end{array}$ \\
\hline Competencias axiológicas & 3 & Creencias, valores y ética. \\
\hline Factores del Entorno & 5 & Económicos, político-legal, tecnológicos, culturales y sociales. \\
\hline
\end{tabular}

Fuente: Elaboración propia

Para establecer el número total de encuestas a realizar se calculó la muestra apoyada en dos bases de datos secundarias oficiales, a saber: 1) Instituto Nacional de Estadística y Censos - INEC y su Directorio Empresarial; 2) Banco Central del Ecuador - BCE, las dos correspondientes al año 2016, de las cuales se extrajo información referente a las actividades económicas de mayor relevancia de las provincias en estudio, para luego realizar un análisis individual de cada una de las provincias con el objetivo de conocer cuáles son los cantones con mayor aporte económico, es así, que las ciudades a encuestar corresponden a las capitales de dichas provincias (Latacunga, Riobamba Puyo y Ambato).

Para esta investigación se ha seleccionado la clasificación de las actividades económicas que realiza el BCE y Clasificación Industrial In- 
ternacional Uniforme - CIIU4.0 quedando así sintetizadas a 14 actividades de las cuales se discriminan 2 de ellas previo al levantamiento de encuestas, como son: administración pública y actividad de suministros de electricidad y agua, dichas actividades no se contemplan como un emprendimiento debido a que son responsabilidad netamente del estado, como se especifica en LOSPEE y LORHUyA $^{6}$.

Para el cálculo de la muestra se ha considerado un muestreo probabilístico estratificado proporcional dentro de cada estrato de las 12 actividades económicas de la zona 3 que pese a ser sectores heterogéneos se contemplan dentro del universo de microempresas de cada provincia.

Para el cálculo de esta población finita se usa el ejemplo 4 del Libro Metodología de la Investigación Científica de Herrera, Medina y Naranjo (2010, pág. 102) en la que se asignan valores: -1 y +1 ya que se desea que el resultado sea más cercano a la realidad, es decir, un valor más exacto.

$$
\begin{array}{|l|l|}
\mathbf{n}=\frac{N}{e^{2}(N-1)+1}=397 & \begin{array}{l}
\text { Dónde: } \\
\text { n: tamaño de la muestra } \\
\text { N: Universo o Población } \\
\text { e: Máximo error admisible } 5 \%
\end{array} \\
\hline
\end{array}
$$

Tabla 8. Datos por provincias para cálculo de la muestra

\begin{tabular}{cccc}
\hline $\begin{array}{c}\text { Ciudades por encuestar } \\
\text { de la zona tres }\end{array}$ & $\begin{array}{c}\text { Número de } \\
\text { Microempresas }\end{array}$ & $\begin{array}{c}\text { Porcentaje de } \\
\text { encuestas a realizarse }\end{array}$ & $\begin{array}{c}\text { Número de encuestas a } \\
\text { realizarse }\end{array}$ \\
\hline Riobamba & 16.694 & $27,29 \%$ & 108 \\
Latacunga & 12.519 & $20,46 \%$ & 81 \\
Puyo & 4.646 & $7,59 \%$ & 30 \\
Ambato & 27.316 & $44,65 \%$ & 177 \\
TOTAL & $\mathbf{6 1 . 1 7 5}$ & $\mathbf{1 0 0 , 0 0 \%}$ & $\mathbf{3 9 7}$ \\
\hline
\end{tabular}

Fuente: Elaboración propia a partir de INEC (2016)

Una vez determinada la muestra correspondiente a la Zona 3 se realiza una distribución porcentual para conocer el número de encuestas a aplicarse en cada una de las provincias, donde de las 397 encuestas 109 corresponden a Chimborazo, 81 a Cotopaxi, 30 a Pastaza y 177 a Tungurahua, distribuidas por cada una de las actividades económicas.

Tras la recolección de los datos se procedió a la tabulación e interpretación de los resultados apoyado en el sistema estadístico SPSS, cuyos datos evidencian la estructura del perfil del emprendedor de la zona 3 basado en sus competencias y motivaciones para emprender.

\section{Resultados}

\section{Características sociodemográficas del emprendedor}

Con los datos extraídos de la encuesta aplicada al estudio, a continuación, se presenta un cuadro comparativo de las cuatro provincias con sus respectivas características sociodemográficas.

Tabla 9. Cuadro comparativo: características sociodemográficas del emprendedor

\begin{tabular}{|c|c|c|c|c|}
\hline $\begin{array}{c}\text { Provincias } \\
\text { Variable }\end{array}$ & Chimborazo & Cotopaxi & Pastaza & Tungurahua \\
\hline Sexo & Hombres (55\%) & Hombres (63\%) & Hombres $(66,7 \%)$ & Hombres (61\%) \\
\hline Edad & $30-40$ años $(28,4)$ & $30-40(35,8 \%)$ & $40-50(33,3)$ & $30-40(28,2)$ \\
\hline Estado civil & Casado $(57,8 \%)$ & Casado $(66,7 \%)$ & Casado $(46,7 \%)$ & Casado $(62,1 \%)$ \\
\hline Número de hijos & Hasta 2 (58,7\%) & Hasta $2(71,6 \%)$ & Hasta $2(56,7)$ & Hasta $2(68,4 \%)$ \\
\hline Nivel de instrucción & Superior $(50,50 \%)$ & superior $(64,2)$ & Superior (50\%) & Superior $(39 \%)$ \\
\hline Sector Económico & Construcción $(21,86)$ & $\begin{array}{l}\text { Agricultura, ganadería, } \\
\text { silvicultura y pesca } \\
(25,23 \%)\end{array}$ & $\begin{array}{c}\text { Explotación de } \\
\text { minas y canteras } \\
(38,63 \%)\end{array}$ & $\begin{array}{c}\text { Manufactura } \\
(14,62 \%)\end{array}$ \\
\hline
\end{tabular}
por provincias de la zona 3

Fuente: Eaboración propia a partir de encuesta y BCE,variables macroeconómicas cantonales (2016-2018)

En la tabla 9 se presenta un cuadro comparativo de las características sociodemográficas de las cuatro provincias de la zona 3, visualizando datos similares a excepción de la edad de los emprendedores, es el caso de Pastaza, donde su edad promedio está entre 40 a 50 años. Otro aspecto diferenciador es el sector económico, pues la construcción mueve la economía en Chimborazo, mientras que para Cotopaxi lo hace la agricultura. Pastaza se diferencia con la explotación de minas y canteras; finalmente, en Tungurahua predomina la manufactura.

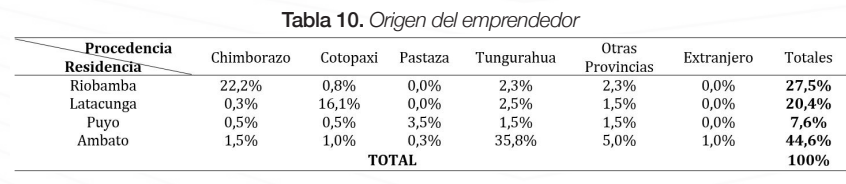

Fuente: Elaboración propia a partir de encuesta (2018)

Se puede percibir que, en la mayoría de las provincias, los emprendedores son nativos de dichas ciudades, excepto en Pastaza, donde mayoritariamente son oriundos de otras provincias, esto representa el $4,1 \%$ del $7,6 \%$ asignado del porcentaje total de la zona encuestada. Sin embargo, el $100 \%$ emprendedores han permanecido en la ciudad donde está su emprendimiento, debido a la oportunidad estratégica que han tenido para crear su empresa y crecer económicamente.

Con los datos tabulados se extrajeron los atributos que deben poseer los emprendedores de las provincias de la zona 3 y poder determinar sus rasgos distintivos. En la tabla 11 se presenta la descripción de las características asociadas al perfil de los emprendedores encuestados.

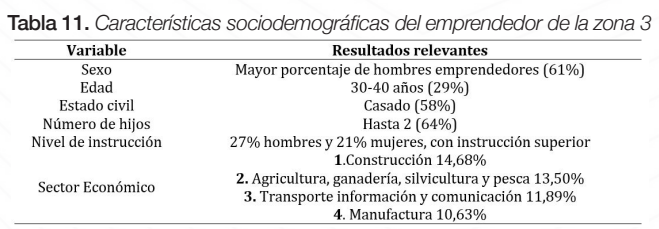

Fuente: Elaboración propia a partir de encuesta (2018)

El mayor porcentaje de emprendedores de la zona 3 del Ecuador está representado por hombres comprendidos entre una edad promedio de 30 a 40 años de estado civil casado con un promedio de 2 hijos. Posee un nivel de instrucción superior. Los emprendimientos se concentran en 4 sectores: Construcción; agricultura, ganadería, silvicultura y pesca; seguido de transporte, información y comunicación; y, manufactura.

\section{Estructura empresarial zona tres}

\begin{tabular}{|c|c|c|c|c|c|c|c|c|c|c|c|}
\hline $\begin{array}{l}\text { Empleados } \\
\text { Cargo }\end{array}$ & 1 & 2 & 3 & 4 & 5 & 6 & 7 & 8 & 9 & 10 & \\
\hline Gerente & $39,0 \%$ & $23,7 \%$ & $9,8 \%$ & $4,3 \%$ & $2,8 \%$ & $1,3 \%$ & $1,0 \%$ & $2,3 \%$ & $0,5 \%$ & $4,0 \%$ & \\
\hline Propietario & $0,3 \%$ & $0,8 \%$ & $0,0 \%$ & $1,0 \%$ & $0,0 \%$ & $0,3 \%$ & $0,5 \%$ & $0,0 \%$ & $0,0 \%$ & $0,8 \%$ & \\
\hline Administrador & $1,0 \%$ & $2,0 \%$ & $1,3 \%$ & $0,3 \%$ & $0,3 \%$ & $0,5 \%$ & $0,0 \%$ & $0,3 \%$ & $0,0 \%$ & $0,3 \%$ & Total \\
\hline Socio & $0,8 \%$ & $0,8 \%$ & $0,3 \%$ & $0,3 \%$ & $0,0 \%$ & $0,0 \%$ & $0,0 \%$ & $0,0 \%$ & $0,0 \%$ & $0,0 \%$ & \\
\hline Total & $41,1 \%$ & $27,2 \%$ & $11,3 \%$ & $5,8 \%$ & $3,0 \%$ & $2,0 \%$ & $1,5 \%$ & $2,5 \%$ & $0,5 \%$ & $5,0 \%$ & $100,0 \%$ \\
\hline
\end{tabular}

Tabla 12. Estructura empresarial según número de empleados de la zona 3 del Ecuador

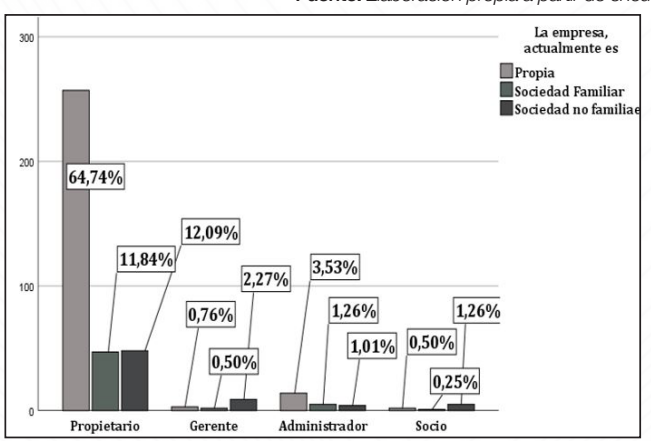

Fuente: Elaboración propia a partir de encuesta (2018) Figura 1. Estructura y cargo empresaria

Los datos de la tabla 12 y figura 1, evidencian que la estructura empresarial de la zona 3 , se concentra en las microempresas formadas por un máximo de 10 empleados, según la clasificación del Instituto Nacional de Estadísticas y Censos (2018, pág. 9). Estas empresas o negocios en un $69 \%$ son administradas por sus propietarios, siendo estos, en su mayoría los únicos que llevan a cargo la activad económica de sus negocios, es decir, que las microempresas de la zona centro son de tipo unipersonales $(41,1 \%)$. Es importante indicar que, de la muestra encuestada, aproximadamente el 17\% corresponden a socie- 
dades no familiares, identificadas dentro de los sectores del transporte e instituciones financieras. Para el caso de las sociedades familiares muestra una representación del 14\% con una presencia mayoritaria de empresas en los sectores de la construcción y enseñanza.

\section{Determinación del perfil del emprendedor a partir del análisis descriptivo univariante}

Tabla 13. Comparativo de las principales Competencias del emprendedor de la zona 3

\begin{tabular}{|c|c|c|c|c|c|c|c|c|}
\hline Provincias & Chimbor: & & Cotopax & & Pastaza & & Tunguraht & \\
\hline$\underbrace{\text { Valoración }}_{\begin{array}{l}\text { Competencias } \\
\text { C.ps individuales }\end{array}}$ & $\begin{array}{c}\text { Muy } \\
\text { importante e } \\
\text { importante }\end{array}$ & Otros & $\begin{array}{c}\text { Muy } \\
\text { importante e } \\
\text { importante }\end{array}$ & Otros & $\begin{array}{c}\text { Muy } \\
\text { importante e } \\
\text { importante }\end{array}$ & otros & $\begin{array}{c}\text { Muy } \\
\text { importante e } \\
\text { importante }\end{array}$ & Otros \\
\hline Motivación al logro & $99,1 \%$ & $0,9 \%$ & $100,0 \%$ & $0,0 \%$ & $100,0 \%$ & $0,0 \%$ & $97,7 \%$ & $2,3 \%$ \\
\hline $\begin{array}{l}\text { Compromiso } \\
\text { Cón }\end{array}$ & $98,2 \%$ & $1,8 \%$ & $100,0 \%$ & $0,0 \%$ & $96,7 \%$ & $3,3 \%$ & $97,2 \%$ & $2,8 \%$ \\
\hline $\begin{array}{c}\text { Orientación a las } \\
\text { oportunidades }\end{array}$ & $98,2 \%$ & $1,8 \%$ & $98,8 \%$ & $1,2 \%$ & - & & - & - \\
\hline Control interno & - & - & - & - & $100,0 \%$ & $0,0 \%$ & - & - \\
\hline $\begin{array}{c}\text { Innovación } \\
\text { C. ps. colectivas }\end{array}$ & - & - & - & - & - & - & $97,7 \%$ & $2,3 \%$ \\
\hline Capacidad comunicación & $99,1 \%$ & $0,9 \%$ & $100,0 \%$ & $0,0 \%$ & $100,0 \%$ & $0.0 \%$ & $98,9 \%$ & $1,1 \%$ \\
\hline Capacidad negociación & $98,2 \%$ & $1,8 \%$ & $96,3 \%$ & $3,7 \%$ & $100,0 \%$ & $0,0 \%$ & $95,5 \%$ & $4,5 \%$ \\
\hline & $96,3 \%$ & $3,7 \%$ & & & $96,7 \%$ & $3,3 \%$ & $98,9 \%$ & $1,1 \%$ \\
\hline $\begin{array}{l}\text { Espiritu de equipo } \\
\text { C. cognitivas }\end{array}$ & & . & $96,3 \%$ & $3,7 \%$ & - & & & \\
\hline Conocimiento en el área & & & & & & & & \\
\hline $\begin{array}{l}\text { específica del negoci } \\
\text { Educción informa }\end{array}$ & $91,7 \%$ & $\begin{array}{l}8,3 \% \\
8,3 \%\end{array}$ & $\begin{array}{l}96,3 \% \\
91,4 \%\end{array}$ & $\begin{array}{l}3,7 \% \\
8,6 \%\end{array}$ & $\begin{array}{l}96,7 \% \\
100 \% \%\end{array}$ & $\begin{array}{l}3,3 \% \\
0,0 \%\end{array}$ & $\begin{array}{l}91,0 \% \\
92,1 \%\end{array}$ & $\begin{array}{l}9,0 \% \\
7,9 \%\end{array}$ \\
\hline $\begin{array}{l}\text { Educación formal } \\
\text { C. axiológicas }\end{array}$ & $82,6 \%$ & $\begin{array}{l}17,4 \% \\
17,4 \%\end{array}$ & $86,4 \%$ & $\begin{array}{l}0,070 \\
13,6 \%\end{array}$ & $86,7 \%$ & $13,3 \%$ & $86,4 \%$ & $13,6 \%$ \\
\hline Ética & $98,2 \%$ & $1,8 \%$ & $98,8 \%$ & $1,2 \%$ & 100 & $0,0 \%$ & $98,7 \%$ & $1,3 \%$ \\
\hline & $97,2 \%$ & $2,8 \%$ & $98,8 \%$ & $1,2 \%$ & $100,0 \%$ & $0,0 \%$ & $98,9 \%$ & $1,1 \%$ \\
\hline $\begin{array}{l}\text { Creencias } \\
\text { Factores del Entorno }\end{array}$ & $76,1 \%$ & $23,9 \%$ & $67,9 \%$ & $32,1 \%$ & $83,3 \%$ & $16,7 \%$ & $73,4 \%$ & $26,6 \%$ \\
\hline Económic & $96,3 \%$ & $3.7 \%$ & $92,6 \%$ & 7.4 & $93,3 \%$ & $6,7 \%$ & $90,4 \%$ & $9,6 \%$ \\
\hline & & & & & & & & \\
\hline Sociales & $89.9 \%$ & $10.1 \%$ & $96,3 \%$ & $37 \%$ & $867 \%$ & $13.3 \%$ & $87.6 \%$ & $124 \%$ \\
\hline
\end{tabular}

Fuente: Elaboración propia a partir de encuesta (2018)

Las competencias de las 4 dimensiones y el grupo de factores externos se muestran en la tabla 13, es así que se puede visualizar las más significativas de cada una de las provincias.

Este cuadro comparativo evidencia que, de las 8 competencias individuales propuestas para este estudio, se seleccionaron los tres atributos más significativos para cada provincia. Para Chimborazo y Cotopaxi, predominan la motivación al logro, el compromiso y orientación a las oportunidades. El emprendedor de la provincia de Pastaza destaca en motivación al logro, compromiso y control interno. En el caso de Tungurahua, las competencias individuales más relevantes son: motivación al logro, compromiso e innovación. Cabe destacar que las competencias comunes son motivación al logro, compromiso, demostrando que el emprendedor del centro el país es un individuo empoderado con su proyecto y su actividad. El tercer atributo cambia en función al sector económico predominante de cada provincia.

Dentro de las competencias colectivas destacan: la capacidad de comunicación, negociación y liderazgo, excepto Cotopaxi, que muestra como tercer atributo relevante el espíritu de equipo.

Con respecto a las competencias cognitivas se observa que existe similitud en las tres características relevantes a esta dimensión, pues estos emprendedores consideran "muy importante e importante" el tener un conocimiento específico del negocio, apoyados en una educación informal y formal para la implementación de sus emprendimientos, es decir, que su experiencia se fortalece a través de cursos, seminarios, talleres, entre otros, así como por conocimientos adquiridos en instituciones de educación superior.

La cuarta dimensión formada por las competencias axiológicas está liderada por la ética como característica importante en las cuatro provincias, seguida de los valores y las creencias, dejando constancia de que el emprendedor de esta zona es una persona que destaca en sus características intrínsecas, necesarias en el desarrollo de sus actividades.

Por otro lado, los factores externos que más afectan a las actividades emprendedoras de la zona 3, corresponden a aquellos de orden económico, tecnológico y social.
Determinación del perfil del emprendedor mediante comprobación de estadígrafos

Para determinar el perfil del emprendedor se puede aplicar un estadístico de validación. Para lo cual se considera aquellas correlaciones con valores $<0,5$ que deben ser discriminados debido a que probablemente no sean muy útiles. Para comprobar si los datos pueden ser correlacionados, se aplica la siguiente formula:

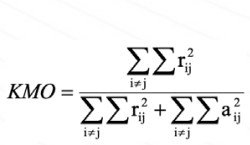

$$
\begin{aligned}
& \text { Dónde: } \\
& \text { rij: coeficiente de correlación lineal de } \\
& \text { Pearson entre las variables i,j } \\
& \text { aij: coeficiente de correlación parcial entre las } \\
& \text { variables i,j }
\end{aligned}
$$

Tabla 14. Comprobación KMO y Prueba Bartlett

\begin{tabular}{|ll|r|}
\hline $\begin{array}{l}\text { Medida de adecuación muestral de } \\
\text { Kaiser-Meyer-Olkin. }\end{array}$ &, 791 \\
Prueba de & Chi-cuadrado \\
esfericidad de & aproximado & 2444,184 \\
Bartlett & gl & \\
& Sig. &, 006 \\
\hline
\end{tabular}

Fuente: Elaboración propia a partir de sistema estadístico SPSS (2018)

En este caso como aceptamos que el modelo de componentes principales nos ayuda a definir el perfil del emprendedor con una significancia de 0,00 y un valor $\mathrm{KMO}$ de 0,79 , esto indica que la definición del perfi del emprendedor se ajusta al estadístico de componentes principales.

Mediante la matriz de correlaciones se evidencia las relaciones más significativas entre las variables de estudio, siendo las más destacadas: las competencias axiológicas, determinadas por la ética y los valores con un 0,727 ; seguida de los factores de entorno, culturales y sociales, representadas con una relación del 0,525. (Matriz de correlaciones ver en anexos)

Para determinar el perfil del emprendedor se apoya en las combinaciones que se muestran en la matriz de correlaciones. Con el análisis factorial ACP se agrupan aquellas que son más relevantes, del 100\% de los resultados, 9 grupos que representan el 57,27\%, permiten establecer el perfil del emprendedor.

La ACP tiene dos condiciones de cumplimiento: 1) los valores de relación entre variables deben ser $>0,5 ; y, 2$ ) el grupo de estudio debe mostrar más de tres variables con valores $>0,5$. Además, si dentro del mismo grupo se muestran más de tres variables en las condiciones descritas, éste será el que mejor especifique las competencias del emprendedor.

En la tabla 15 se muestran los tres de los nueve grupos más significativos, el primero, valida el perfil del emprendedor, debido a que cumple con los criterios de correlación y análisis factorial

Tabla 15. Análisis de componentes principales

\begin{tabular}{cccc}
\hline & \multicolumn{3}{c}{ COMPONENTES } \\
\cline { 2 - 4 } Asumir riesgos & 1 & 2 & 3 \\
Autonomía &, 359 &, 196 &,- 378 \\
Compromiso &, 387 &, 249 &,- 265 \\
Control interno &, 449 &, 172 &,- 130 \\
Innovación &, 468 &, 168 &,- 182 \\
Motivación al logro &, 591 &, 174 &,- 161 \\
Orientación a las oportunidades &, 515 &, 240 &,- 063 \\
Tolerancia a la ambigüedad &, 500 &,- 095 &,- 040 \\
Capacidad de comunicación &, 549 &,- 002 &,- 142 \\
Capacidad de negociación &, 459 &, 170 &,- 187 \\
Espíritu de equipo &, 448 &,- 008 &, 006 \\
Liderazgo &, 390 &, 393 &, 437 \\
Planificación &, 495 &, 408 &, 105 \\
Educación formal &, 406 &, 274 &, 407 \\
Educación informal &, 347 &,- 034 &, 165 \\
Experiencia previa &, 291 &,- 154 &, 288 \\
Creencias &, 419 &,- 204 &, 148 \\
Valores &, 342 &,- 281 &, 355 \\
Ética &, 315 &,- 361 &,- 184 \\
Económicos &, 535 &,- 008 &,- 386 \\
Políticos-Legales &, 529 &, 073 &,- 354 \\
Tecnológicos &, 458 &,- 269 &, 012 \\
Culturales &, 400 &,- 311 &, 379 \\
Sociales &, 562 &,- 189 &, 129 \\
Sonien &, 510 &,- 487 &, 118 \\
Euento &, 450 &,- 392 &, 248 \\
\hline
\end{tabular}

Fuente: Elaboración propia a partir de encuesta y sistema estadístico SPSS (2018) 
Tras la aplicación de los estadígrafos se comprueba que el perfil del emprendedor de la zona 3 del Ecuador está determinado por las competencias psicológicas individuales con sus características de: control interno, innovación, motivación al logro, orientación a las oportunidades y la tolerancia a la ambigüedad. En las competencias psicológicas colectivas se consideran la capacidad de comunicación y liderazgo. En el caso de las competencias axiológicas se contemplan la ética y los valores. Para el grupo de factores del entorno que enfrentan los emprendedores son los de orden económico, tecnológi$\mathrm{co}$, cultural y social.

\section{Conclusiones}

Para poder formular el perfil de un emprendedor es necesario conocer las características que poseen estas personas, desde un aspecto sociodemográfico se evidencia que los emprendedores de la zona 3 están liderados por los hombres, con una estructura familiar definida, este rasgo deja ver que la responsabilidad de un hogar genera una fortaleza que les lleva a considerar la idea de trabajar de manera independiente creando sus propios negocios o empresas, por medio del impulso de sus familias.

Otro dato relevante de los aspectos sociodemográficos, destaca que los emprendedores encuestados tienen un nivel de instrucción superior, esto puede ayudar a consolidar las empresas, así como garantizar su supervivencia. Un porcentaje mayoritario de encuestados manifiestan que la capacitación continua afianza sus conocimientos específicos para resolver necesidades empresariales.

En relación a las actividades económicas que mueven a la zona 3, de acuerdo al Banco Central del Ecuador BCE y validadas con la información recogida en la encuesta, el sector de la construcción aporta el mayor PIB de crecimiento económico del centro del país, seguido de la agricultura, el transporte y la manufactura, debido a que las 4 provincias se encuentran en un espacio geográfico privilegiado, para comunicarse con otras regiones del Ecuador.

Este estudio se ha enfocado en las microempresas y se puede determinar que la estructura de éstas en su mayoría es unipersonales, administradas por sus propietarios, esto debido a que son negocios que no requieren de muchos empleados para el desarrollo de sus actividades.

Además, la mayoría de los emprendedores establecieron sus empresas en su ciudad natal a excepción de Pastaza donde los emprendedores son oriundos de otras provincias del país.

El perfil del emprendedor de la zona 3, es un hombre de entre 30 y 40 años, estado civil casado, con dos cargas familiares, instrucción superior, las competencias que destacan a este individuo se reflejan en las siguientes características: control interno, innovación, motivación al logro, orientación a las oportunidades y la tolerancia a la ambigüedad, atributos con los que potencia su capacidad de comunicación y liderazgo, con un nivel elevado de ética y valores morales muy bien definidos. Esta combinación de características, hace del emprendedor de la zona tres, un sujeto, capaz de enfrentar las dificultades de su entorno, principalmente aquellos de orden económico, tecnológico, cultural y social.

Finalmente, esta investigación ha dado apertura a futuras líneas de investigación con respecto a analizar la estructura que tienen los emprendimientos de la zona centro, las circunstancias socio políticas como inconveniente para emprender, las motivaciones que tiene un emprendedor y el éxito o fracaso de los mismos a la hora de iniciar un negocio entre otros temas.

\section{Referencias}

Ahumada Méndez, L. S. (2013). Las Competencias Aplicadas al Emprendimiento. Escenarios, vol 11, Na 1, 44-56. Obtenido de file:///C:/Users/ Biblio-PC12/Downloads/Dialnet-LasCompetenciasAplicadasAIEmprendimiento-4714359.pdf

Albán Navarro, A. D., Batista Hernández, N., Real Zumba, G., \& Valcárcel Izquierdo, N. (2017). Desarrollo de la competencia de emprendimiento; una necesidad en la formación integral del estudiante. Dilemas Contemporáneos:
Educación, Política y Valores, 16.

Alderete, M. V. (2013). Do information and communication technology access and innovation increase outsourcing in small and medium enterprises? Jistem: Journal of Information Systems and Technology Management, vol. 10, núm. 2,, 303-322. Recuperado el 12 de 12 de 2018, de https://www.redalyc.org/ articulo. oa?id=203227937008

Alsinet Mora, C., Paris Mañas, G., Torrelles Nadal, C., \& Sabrià Bernadó, B. (2015). Assessing teamwork competence. Psicothema, vol. 27, núm. 4, 354-361. Recuperado el 12 de 12 de 2018, de https://www.redalyc.org/articulo. oa?id $=72742305007$

Alvarado Mejía, M., Rangel Lúquez, O., \& Sierra Ortiz, E. (2016). El perfil emprendedor como catalizador de la innovación en los jóvenes del departamento de la Guajira. Omnia, vol. 22, núm. 3, 71-86. Obtenido de http://www.redalyc. org/pdf/737/73752819006.pdf

Álvarez, B., Pellicer, C. \& Torrejón, J. L. (2013). Aprender a emprender: como educar el talento emprendedor. España: Centro Editor PDA S.L Grupo Planeta. Obtenido de https://en.fpdgi.org/upload/projecte/aprender-a-emprenderesp.pdf

Amaral, D. J., Hashimoto, M., \& Jorge Nassif, V. M. (2014). Entrepreneurs self-perception of planning skills: evidences from brazilian entrepreneurs. Revista Ibero Americana de Estratégia, vol. 13, núm. 4,, 107-121. Obtenido de http://www.redalyc. org/articulo.oa?id=331237822009

Apetrei, A., Ribeiro, D. Roig, S., \& Mas Tur. A. (Agosto de 2013). E emprendedor social - una explicación intercultural. CIRIEC, 37-52. Obtenido de http://www.redalyc.org/articulo.oa?id=17429865004

Balbastre Benavent, F., Canet Giner, M. T., Escribá Carda, N., \& Ugalde Binda, N. (2014). The Role of Intellectual Capital and Entrepreneurial Characteristics as Innovation Drivers. INNOVAR. Revista de Ciencias Administrativas y Sociales, vol. 24, núm. 53, 41-60. Obtenido de http://www.redalyc.org/articulo. oa?id=81831420004

Benatti, G., \& Saltorato, P. (2016). The organizational restructuring performative act under shareholder value management ideology. Revista Brasileira de Gestión de negocios, 34. Recuperado el 12 de 12 de 2018, de https://www. redalyc.org/jatsRepo/947/94750556006/index.html

Bernal, M. C., Carvalho, C., Mortan, R. A., \& Ripoll, P. (2014). Effects of emotional intelligence on entrepreneurial intention and self-efficacy. Revista de Psicología del Trabajo y de las Organizaciones, vol. 30, núm. 3, 97-104.

Berríos Lugo, J. E. (2017). Levels of Entrepreneurial Traits of University Students in Puerto Rico. Forum Empresarial, vol. 22, núm. 2, 85-108.

Biedma Ferrer, J. M., Martínez Fierro, S., \& Ruiz Navarro, J. (2015). Las condiciones del entorno emprendedor y el desarrollo económico: un análisis de los países GEM. Revista de Economía Mundial, núm. 41, 181-212. Obtenido de http://www.redalyc.org/articulo.oa?id $=86643415008$

Bigio Schnaider, P. S., Cavalcanti Rocha Martins, A., \& Macchione Saes, M. S. (2013). Entrepreneurial decision-making using the knightian uncertainty approach. Revista de Administração - RAUSP, vol. 48, núm. 4, 716-726. Obtenido de http://www. redalyc. org/articulo. oa?id=223429367007

Bonilla Ricardo, R. F., \& García Restrepo, J. O. (2014). Caracterización del perfil emprendedor en graduados de la maestría en administración de negocios: un análisis empírico en la ciudad de Manizales. Manizales.

Caetano, A., Curral , L., \& Santos, S. C. (2013). Psychosocial aspects of entrepreneurial potential. Journal of Small Business \& Entrepreneurship, 661-685. Obtenido de http://repositorio.ul.pt/bitstream/10451/11048/1/5-Santos,\%20 Caetano\%20\%26\%20Curral,\%202014.pdf

CAF Banco de desarrollo de América Latina. (2016). Más habilidades para el trabajo y la vida: los aportes de la familia, la escuela, el entorno y el mundo laboral. Reporte de Económia y Desarrollo (RED), 231.

Capilla, R. M. (2016). Habilidades cognitivas y aprendizaje significativo de la adición y sustracción de fracciones comunes. Cuadernos de Investigación Educativa, vol. 7, núm. 2, 49-62. doi:https://doi.org/10.18861/cied.2016.7.2.2610 Castañeda Rivas, M., González, M., \& Rubio Royo, E. (2015). Necesidad de formación del perfil del e-emprendedor en la hiper red social: nuevo entorno vital, expandido y complejo. Multiciencias, vol. 15, núm. 4, 452-460. Obtenido de http:// www. redalyc. org/articulo. oa?id=90448465012

Chaturvedi, P. (2014). Axiological competences: The role of teacher training programme in strengthens it. IOSR Journal of Research \& Method in Education (IOSR-JRME), 19-23. Obtenido de http://www.iosriournals.org/iosr-jrme/ papers/Vol-4\%20lssue-2/Version-4/D04241923.pdf

Climént Bonilla, J. B. (2012). El significado de los valores en las competencias individuales y colectivas. Revista Mexicana de Agronegocios, vol. 31 31-41. Obtenido de http://www.redalyc.org/pdf/141/14123108005.pdf

Climént Bonilla, J. B. (2017). Fortalezas y debilidades del concepto polisémico de "competencias". Revista Actualidades Investigativas en Educacion, 1-30. doi:http://dx.doi.org/10.15517/aie.v17i3.29065

Contreras Torres, F., Espinosa Mendez, J. C., Jauregui Machuca, K. Omaña Guerrero , J. A., Portalanza Chavarría, A., \& Soria Barreto , K. (2017). Exploring entrepreneurial intentions in Latin American university students. International Journal of Psychological Research, vol. 10, núm. 2, 46-59. Obtenido de http://www.redalyc.org/articulo.oa?id=299052071006 
Criado, N., Denegri Coria, M., Mendoza, J., Orellana Calderón, L., Salazar, P., Sepúlveda Maldonado, J., \& Yung, G. (2017). Características emprendedoras personales y alfabetización económica: una comparación entre estudiantes universitarios del sur de Chile. Interdisciplinaria, vol. 34, núm. 1, 107124. Obtenido de $h t t p: / / w w w . r e d a l y c . o r g / p d f / 180 / 18052925007 . p d f$

Da Silva Faia, V., Domingues da Silva, J., \& Vier Machado, H. P. (2016). Entrepreneurial alertness: Study of the Influence of Individual Characteristics and Entrepreneurship. BBR - Brazilian Business Review, vol. 13, núm. 5, 85-107. Obtenido de $h$ ttp://www.redalyc.org/articulo.oa?id=123047026004

Davidsson, P., Obschonka, M. Stuetzer, M., \& Schmitt-Rodermund, E. (2013). Where do entrepreneurial skills come from? Applied Economics Letters, 10. Obtenido de https://mpra.ub.uni-muenchen.de/48274/1/MPRA_paper 48274.pdf

De Azevedo Ávila, H., da Rocha, A., \& Ferreira da Silva, J. (2015). Brazilian Multinationals' Ownership Mode: The Influence of Institutional Factors and Firm Characteristics. BAR - Brazilian Administration Review, vol. 12, núm. 2, 190-208. Recuperado el 12 de 12 de 2018, de https://www.redalyc.org/articulo. oa?id=84139453004

De Souza Libânio, C., \& Gonçalves Amaral, F. (2016). Competencies and Interactions in Design Management. BBR - Brazilian Business Review, vol. 13, núm. 6, 191-209.

De Souza Sant'Anna, A., \& Martins Diniz, D. (2017). (Re-)construing Space as Capital: Contributions from a Study with Local Entrepreneurs. BBR Brazilian Business Review, vol. 14, núm. 5, 544-558. Recuperado el 12 de 12 de 2018, de https://www. redalyc.org/articulo.oa?id=123052461006

Dion, M. (2014). The economic and non-economic dimentions of social entreprises moral discouses: an issue of axiological and philosopjical coherence. Int. Entrep. Manag. J., 385-408.

Dorado Urbano, J. E., Pineda Zapata, U., \& Morales Gualdrón, S. T. (2014). Factores del servicio y del perfil del emprendedor que inciden en la superviviencia empresarial: una revisión crítica. LASALLISTA, 181-194. Obtenido de http://www.redalyc.org/articulo.oa?id=69539788020

Dos Santos Fabricio, J., Gazolo, S., Moreno Anez, M. E., \& Vier Machado, H. P. (2016). Women entrepreneurs: reasons and difficulties for starting in business. RAM. Revista de Administração Mackenzie, vol. 17, núm. 3, 15-38. Recuperado el 12 de 12 de 2018, de https://www.redalyc.org/articulo. oa?id=195446414002

Duarte Masi, S., \& Sung Park, S. I. (2015). El perfil del emprendedor y los estudios relacionados a los emprendedores Iberoamericanos. Revista Internacional de Investigación Siencias Sociales, 291-314. Obtenido de http://scielo. iics.una.py/pdf/riics/v11n2/v11n2a10.pdf

Edgerton, J. D., \& Roberts, L. W. (2014). En ".f.achievement", Encyclopedia of Quality of Life and well-Being Research. Springer Nethearlands.

Feitó Madrigal, D., Mungaray Lagarda, A., \& Texis-Flores, M. (2016). Factors associated with learning management in Mexican micro-entrepreneurs. ICESI, 15. doi:10.1016/i.estger.2016.10.003

Ferreira, S. \& Jolonch, X. (30 de Octubre de 2015). ¿De qué están hechos nuestros emprendedores? Redalyc. doi:http://dx.doi.org/10.12804/rev. univ.empresa.30.2016.09

Florez, J. L., Hernández, B., Sánchez García, J. C. \& Ward, A. (2017). Educación emprendedora: Estado del arte. Propósitos y Representaciones vol. 5. N 2 Scielo, 401-473. Obtenido de http://www.scielo.org.pe/pdf/pyr/v5n2/ a10v5n2.pdf

Fuentelsaz, L., \& Montero, J. (2015). ¿Qué hace que algunos emprendedores sean más innovadores? UNIVERSIA BusinessReview, 14-31. Obtenido de http://www.redalyc.org/articulo.oa?id=43341001001

Gámez Gutiérrez, J., Saiz Álvarez, J. M. \& Gil Ángel, G. (2016). A cognitive, Emotional and Behavioral Assessment of Colombian Entrepreneurs Attitudes Toward Corruption. Universidad \& Empresa, 38. doi:10.12804/revistas. urosario. edu.co/empresa/a.4682

Gil Gaytán, O. L. (2017). Asociación entre el perfil del empresario y el éxito en el emprendimiento internacional. Paakat: Revista de Tecnología y Sociedad, 17.

Godelieve Van den Brande, Y. P., \& Panagiotis Kampylis, M. B. (2016). EntreComp: The Entrepreneurship Competence Framework. Luxembourg: Join Research Center.

González Campo, C. H., Murillo Vargas, G., \& Osorio Tinoco, F. (2016). El Emprendedor y el Ejercicio del poder en el Entorno Institucional. Scielo, 53. Obtenido de http://www.scielo.org.co/pdf/diem/v14n2/v14n2a04.pdf

Gutiérrez Olvera, S. (2015). Emprendimiento en las empresas. Revista Iberoamericana de Contaduría, Economía y Administración, 19 Vol. 4, Núm. 7. Obtenido de file:///C:/Users/Biblioteca/Desktop/Downloads/Dialnet-EmprendimientoEnLasEmpresasFamiliares-5294260.pdf

Guzmán Barquet, E. A., Sopó Montero, G. R., Salazar Raymond, M. B., \& Vera Salas, L. G. (2017). Liderazgo como competencia emprendedora. ESPACIOS, 24. Obtenido de http://www.revistaespacios.com/a17v38n24/ a17v38n24p24.pdf

Herrera Guerra, C. E. (2012). Una investigación en emprendimiento: caracterización del emprendedor. Económicas CUC, 191-204. Obtenido de
file:///C:/Users/Biblioteca/Desktop/Downloads/Dialnet-UnalnvestigacionEnEmprendimiento-5085553.pdf

Herrera Guerra, C. E., \& Montoya Restrepo, L. A. (2013). El emprendedor: una aproximación a su definición y caracterización. Dialnet, 22.

Hidalgo Proaño, L. F. (2014). La cultura del emprendimiento y su formacion. Alternativas UCSG, 46-50. Obtenido de file:///C:/Users/Biblioteca/Desktop/ Downloads/Dialnet-LaCulturaDelEmprendimientoYSuFormacion-5599803.pdf Hnátek, M. (2015). Entrepreneurial thinking as a key factor of family business success. Procedia - Social and Behavioral Sciences, 342-348. Hochsztain, E., \& Messina , M. (2015). Factores de Éxito de un emprendimiento: un estudio exploratorio con base en técnicas de Data Mining. Tec Empresarial, 31-40.

Ina Primiana, S. I. (2015). Internal And External Environment Analysis On The Performance Of Small And Medium Industries (Smes) In Indonesia. International Journal Of Scientific \& Technology Research Volume 4, 9.

Instituto Nacional de Estadisticas y Censos. (2018). Encuesta estructural empresarial ENESEM 2016. Ecuador: Ecuadorencifras.

Jiménez Leube, J. (2017). Perfil del emprendedor y fomento del emprendimiento: un enfoque personalista. REVISTA DE ESTUDIOS DE JUVENTUD, 16. Obtenido de $h$ ttp://www.injuve.es/sites/default/files/2017/46/publicaciones/ revista107_10-perfil-del-emprendedor-y-fomento-del-emprendimiento.pdf

Klein, P. G. (2016). Why entrepreneurs need firms, and the theory of the firm needs entrepreneurship theory. Revista de Administração - RAUSP, vol. 51 , núm. 3, 323-326. Recuperado el 12 de 12 de 2018, de https://www.redalyc.org/ articulo. oa?id=223446848009

Ley Orgánica de Recursos Hídricos, Usos y Aprovechamiendo del Agua. (6 de Agosto de 2014). Lexis. Obtenido de Lexis: https://www.agua.gob. ec/wp-content/uploads/2012/10/LEYD-E-RECURSOS-HIDRICOS-II-SUPLEMENTO-RO-305-6-08-204.pdt

Ley Organica del Servicio Publico de Energia Electrica. (16 de Enero de 2015). Lexis. Obtenido de Lexis: https://www.iner.gob.ec/wp-content/uploads/ downloads/2015/05/LOSPEE.pdf

Lizote, S. A., Silveira, A., \& Verdinelli, M. A. (2013). Organizational factors and intrapreneurial competences. International Journal of Innovation, vol. 1, núm. 1, 1-12. Recuperado el 12 de 12 de 2018, de https://www.redalyc.org/articulo. oa?id $=499150950001$

López Cózar Navarro, C., Lupiáñez Carrillo, L., \& Priede Bergamini, T. (2014). El emprendimiento como motor del crecimiento económico. Boletín Económico DE ICE No 3048, 10

López Gómez, E. (2016). En torno al concepto de competencia: un análisis. Revista de Currículum y Formación de Profesorado, vol. 20, núm. 1, 311 - 322. Obtenido de http://www.redalyc.org/pdf/567/56745576016.pdf

López Pérez, M. I., Lujano Montiel, J., \& Ortega Luzardo, A. T. (2015). Características emprendedoras de los gerentes de las empresas fabiliares de la poblacion de Santa Bárbara de Zulia, Venezuela. Visión Gerencial, núm. 1, 139164. Obtenido de $h t t p: / / w w w . r e d a l y c$. org/articulo. oa?id=465545898007

Lupiáñez Carrillo, L., Priede Bergamini, T. \& López-Cózar Navarro, C. (2014). El emprendimiento como motor del crecimiento económico . Boletín Económico de ICE N 3048, 10.

Martínez Gómez, A. E. (2016). Factores socio-culturales asociados al emprendedor: evidencia empírica para América Latina. Revista Venezolana de Gerencia, 312-330. Obtenido de http://www.redalyc.org/articulo. oa?id=29046685009

Marulanda Valenia, F. Á., Montoya Restrepo, I. A., \& Vélez Restrepo, J. M. (2014). Aportes teóricos y empíricos al estudio del emprendedor. Scielo, 11.

Meza Flores, I. C. (2013). Entre el conocimiento formal e informal: esfuerzos interinstitucionales para la inclusión en el aprendizaje. Revista Interamericana de Educación de Adultos, vol. 35, núm. 1, 8-21.

Montiel Campos, H., \& Palma Chorres, Y. M. (2016). Technological Entrepreneurship: A Multilevel Study. Journal of Technology Management \& Innovation, vol. 11, núm. 3, 77-83. Obtenido de http://www.redalyc.org/articulo. oa?id $=84748406009$

Morales Gualdrón, S. T., \& Pineda Zapata, U. (2015). Factores del perfil del emprendedor y de la gestión del servicio que inciden en la supervivencia empresarial: casos del oriente de Antioquia (Colombia). Pensamiento \& Gestión, núm. 38, 176-207. Obtenido de http://www.redalyc.org/articulo. oa?id=64639792010 Nandram, S. S., Orhei, L. E., \& Vinke, J. (2015). Social entrepreneurship competence: evidence from founders of social enterprises in Romania. Int. J. Entrepreneurship and Small Business, Vol. 25, No. 1, 80-105. Obtenido de file:///C:/ Users/Biblio-PC10/Downloads/IJESB250105ORHEl.pdf

Parra Miranda, M. S. (2017). El proceso creativo y emprendedor. Instituto de Tecnologías Sudamericano, 18. Obtenido de file:///C:/Users/Biblio-PC75/ Downloads/Parra2017EIProcesoCreativoyEmprendedor.pdf

Pedrosa, I., \& Suárez Álvarez , J. (2016). The assessment of entrepreneurial personality: The current situation and future directions. Papeles del Psicólogo, 2016. Vol. 37(1), 62-68. Obtenido de file:/l/C:/Users/Biblio-PC10/ Downloads/evaluacindelapersonalidademprendedora. situacinactualy/neasdefuturo English Papelespsiclogo 161.pdf

Restrepo Salgado, E., Tapasco Triviño, Ó. J., \& Vidarte Claros, J. A. 
(2016). Perfil emprendedor de estudiantes de la Tecnología en gestión agropecuaria, Universidad de Caldas. Ánfora, 87-106. Obtenido de http://www.redalyc. org/articulo.oa?id $=357848839004$

Rivera Badillo, P. L. (2016). El emprendimiento y la creación de empresa. Etapas y factores clave de éxito. Madrid.

Rivera Badillo, P. L. (2017). Competencias Esenciales del Emprendedor. Ambato: Consejo Editorial Universotario.

Rodionova, V. I., \& Shvachkina, L. A. (2018). Intercultural Communications as a Tool of Formation of the Global Axiological System in the Conditions of Establishment of the Entrepreneurial Type of Thinking of the Russian Society. Journal of History Culture and Art Research, 452-459.

Romero Carrasquero, Y., \& Tapia Luzardo, F. (2014). Desarrollo de las habilidades cognitivas en niños de edad escolar. Multiciencias, vol. 14, núm. 3, 297-303. Obtenido de http://www.redalyc.org/pdf/904/90432809008.pdf

Viloria, H. (2017). Desarrollo de competencias emprendedoras en docentes de universidades públicas autónomas. Revista Encuentros, 133-146. doi:http://dx.doi.org/10.15665/re.v15i1.815 